\title{
CONSUBSTANTIALITÉ DU CANAL ET DE L'ÉNONCIATION : LE CAS DU GRAFFITI, INSCRIPTION SUPERPOSÉE ET MARGINALE
}

\author{
Adrien Mathy
}

L'Harmattan | «Cahiers internationaux de sociolinguistique »

2019/1 N 15 | pages 89 à 120

ISSN 2257-6517

ISBN 9782343182056

Article disponible en ligne à l'adresse :

https://www.cairn.inforevue-cahiers-internationaux-desociolinguistique-2019-1-page-89.htm

Distribution électronique Cairn.info pour L'Harmattan.

(C) L'Harmattan. Tous droits réservés pour tous pays.

La reproduction ou représentation de cet article, notamment par photocopie, n'est autorisée que dans les limites des conditions générales d'utilisation du site ou, le cas échéant, des conditions générales de la licence souscrite par votre établissement. Toute autre reproduction ou représentation, en tout ou partie, sous quelque forme et de quelque manière que ce soit, est interdite sauf accord préalable et écrit de l'éditeur, en dehors des cas prévus par la législation en vigueur en France. Il est précisé que son stockage dans une base de données est également interdit. 


\section{CONSUBSTANTIALITÉ DU CANAL ET DE L'ÉNONCIATION : LE CAS DU GRAFFITI, INSCRIPTION SUPERPOSÉE ET MARGINALE ${ }^{1}$}

\section{PROLÉGOMÈNES}

\section{Introduction}

Souvent cantonnée à une étude sociolinguistique (en témoignent les nombreuses occurrences des termes parler jeune, urbanité, etc., dans les études sur les graffitis ${ }^{2}$ ), la pratique du graffiti ne dispose d'aucun appareil conceptuel adéquat qui permettrait de la définir et de l'appréhender dans toute sa réalité langagière. S'il est vrai que de nombreuses études ont été consacrées à cette pratique - études que nous exposerons brièvement dans notre état de l'art -, peu d'entre elles cherchent effectivement à définir le graffiti dans ce qu'il aurait de linguistiquement singulier. Toutefois, compte tenu de la position cardinale du graffiti au sein des études sociolinguistiques, il nous paraît nécessaire de définir précisément sa nature et ses conditions de réalisation afin d'analyser les facteurs qui en font une forme énonciative idoine à l'expression de contenus socialement marqués.

Il nous semble nécessaire de concevoir un appareil conceptuel et d'établir une terminologie ad hoc, afin de construire ensuite une typologie souple et économique, organisant notre relevé empirique, étayé par l'outil statistique. Notre objectif est effectivement de proposer une analyse qui mettrait au jour la singularité de l'énoncé graffiti et de son énonciation; singularité, par ailleurs, porteuse des nombreuses interrogations appréhendées par la pragmatique et la praxématique. Nous nous sommes alors appliqué, à travers notre étude, à rendre compte de ces interrogations, via la dialectique entre une démarche théorique et empirique, tout en instaurant un dialogue entre la théorie linguistique de l'énonciation et les nombreuses approches qui ont déjà proposé des analyses du phénomène, fussent-elles anthropologiques, sociologiques, ou du domaine des sciences cognitives.

Cet appareil conceptuel, ainsi que la typologie qui lui est associée, est évidemment construit sur base de l'analyse d'un corpus. Cette construction est divisée en deux parties. La première partie consiste en un liminaire théorique, nécessaire à la circonscription de toute l'empirie du phénomène. Aux définitions naïves, il nous faut substituer une définition capable d'organiser et

\footnotetext{
${ }^{1}$ Adrien Mathy, Université de Liège, Belgique, amathy@student.ulg.ac.be

${ }^{2}$ Plusieurs études exemplifiant cette tendance sont rapportées dans l'état de l'art.
} 
de trier les éléments constituant notre corpus de sorte à pouvoir ensuite l'analyser avec pertinence. Nous devons toutefois aborder la résolution d'une difficulté heuristique afin de comprendre la prégnance de l'appareil conceptuel. Le corpus étant la base de notre étude, il a été établi antérieurement à l'analyse qui a conduit à une définition du graffiti. Dès lors, le corpus original est constitué selon la définition ordinaire du graffiti. Nous avons donc ensuite opéré une réduction du corpus, sur base de notre définition. La seconde partie de notre analyse repose sur ce corpus réduit. Il s'agit de construire une typologie capable de distinguer avec pertinence différents types de graffitis dont les divergences rendent compte de la pluralité du phénomène étudié. À cette dialectique méthodologique s'ajoute une heuristique particulière. En effet, l'hétérogénéité de notre objet nous oblige à établir une méthode adaptée. En le modélisant, nous risquons de gommer certaines de ses spécificités et de proposer un appareil théorique trop homogénéisant ou émaillé d'exceptions. Aussi, tous nos postulats auront valeur d'hypothèses provisoires dont la validité sera examinée au fil du dialogue entre théorie et empirie.

Le corpus en question a été recueilli dans les bâtiments de l'université de Liège (ULG). Face à l'impossibilité, en termes de moyens, de réaliser une base de données contenant tous les graffitis présents au sein de l'université, nous avons opté pour une sélection de lieux propices à l'activité énonciative dont le graffiti est le résultat - de sorte à ne pas sélectionner arbitrairement les lieux où les graffitis sont présents. Nous avons préféré un conditionnement du corpus - couplé à une connaissance dudit conditionnement - à une apparente exhaustivité qui cacherait un biais de sélection arbitraire. Pour ses caractéristiques particulières en lien avec le phénomène étudié, nous avons sélectionné les toilettes des hommes dans le but d'établir notre corpus. Bien que cette méthode puisse nuire à une généralisation du propos, elle nous permet a contrario d'établir en quoi le lieu conditionne l'énonciation ellemême, et d'interroger l'influence du contexte d'énonciation sur la nature de l'énonciation, intégrant ainsi à notre problématique linguistique des perspectives anthropologiques et sociologiques.

\section{État de l'art et perspectives}

Avant d'aborder la construction de notre appareil théorique, il convient d'exposer l'état de l'art et les perspectives d'étude possibles, afin de positionner notre approche dans sa particularité, partant que le graffiti suscite l'intérêt de nombreux domaines. Nous insistons par ailleurs sur l'impératif de fournir une définition commune exempte d'appréciations idéologiques: le graffiti étant un objet hybride, dépassant sa nature strictement langagière, les approches le concernant se multiplient et mêlent des perspectives 
épistémologiques parfois contradictoires, voire problématiques. Le panorama réalisé par Mensch (2013) dans son état des lieux nous permet de réaliser l'ampleur du défi épistémologique et l'intérêt de notre approche : «Entre une vision du graffiti comme relevant du vandalisme, déployée par Alain Vulbeau, ou une vision du graffiti comme champ de création artistique, défendue par l'avocat Emmanuel Moyne, force est de constater que d'importantes contradictions animaient les débats ${ }^{1} »$. Cette dichotomie entretenue entre une perception du graffiti vandale et du graffiti artistique oblige à proposer une définition qui dépasse ces objections et qui soit strictement empirique, c'est-àdire exempte des perceptions normatives.

Le graffiti ne relevant évidemment pas exclusivement du domaine verbal, il n'est guère étonnant de rencontrer de nombreuses études sémiotiques s'intéressant à la nature graphique ou symbolique de ce dernier, comme en témoignent les travaux de Spinelli (2007), Dávila (2009) ou encore Derycke (2003), etc. De plus, outre l'aspect non verbal du graffiti, la sémiotique a longuement étudié le rapport symbolique qu'il entretient avec l'espace où l'acte a été réalisé - l'aspect intrusif du graffiti a ainsi permis de le rapprocher de la publicité (Dávila, 2009). Bien que nous nous cantonnions à une définition verbale du graffiti, nous accorderons une attention particulière à sa valeur sémiotique et à son ancrage dans l'espace.

Il est en effet intéressant de constater que l'approche sémiologique, parce qu'elle considère la symbolique du phénomène en lien avec celle de l'espace où il s'inscrit, implique la perception socialement construite du récepteur et, conséquemment, ouvre la voie à une perspective sociologique qui interroge les raisons pour lesquelles le graffiti est lieu d'expression idéal pour des contenus (ou de formes) socialement illégitimes. L'étude de ces contenus est par ailleurs l'approche privilégiée par la sociolinguistique: nous pouvons nous appuyer sur les travaux de Billiez (1998), Bloch-Raymond (2002), Felloneau et Busquet (2001), Boudinet (2003), Calo (2003), Ouaras (2009 ; 2015), Bertoncini (2010), etc. La constante, nous l'avons dit, est de considérer le graffiti comme un objet relevant d'une culture jeune, urbaine, voire métissée. L'étude du contenu se fait donc au détriment du contenant et semble oublier la majorité de la production de graffiti qui est celle de l'individu locuteur lambda.

Nous pouvons remarquer que la sociolinguistique est effectivement concentrée sur le contenu du graffiti et sur les raisons de la pratique, confinant parfois au jugement de valeur. Les notions de vandalisme et de création artistique sont ainsi éminemment critiquables tant elles relèvent de la

${ }^{1}$ Il s'agit du compte rendu d'un colloque organisé en 2003 à Bordeaux, dont les communications ont été réunies dans un ouvrage sous la direction de Lecomte (2003), rapporté dans Mensch $(2013,15)$. 
perception du locuteur. De plus, chaque domaine s'en préoccupe comme intermédiaire et non selon sa nature ${ }^{1}$. Toutefois, étudier le graffiti pour luimême, comme nous le proposons, suppose de le considérer comme une pratique singulière située dans le complexe de représentations et de perceptions qu'en a la société : à savoir les fantasmes véhiculés par cette perception, le jugement normatif lié au cadre légal ainsi que l'aspect coercitif et statutaire d'une pratique qui s'institue socialement et artistiquement. Ignorer ces aspects reviendrait à séparer le fait linguistique de la réalité dont il émerge - il est donc nécessaire de ne pas perdre de vue les positions des domaines suscités qui permettent de situer le phénomène dans ladite réalité : réalité de sa nature symbolique, sociétale, historique, psychologique, somme toute anthropologique.

Par ailleurs, bien que notre définition du graffiti se veuille strictement linguistique dans son énonciation, nous ne pouvons évacuer sa dimension anthropologique et sociologique, qui sera approfondie ensuite, lorsqu'il s'agira d'articuler le rapport étroit et complexe entre l'énonciation et les éléments environnementaux propres à son contexte. De ce fait, l'étude de ce phénomène demande une définition stricte et précise qui évacue toutes les représentations stéréotypées. Il faut en outre écarter les pratiques multiples ou trop complexes qui nuiraient à une analyse pertinente du graffiti. Pour cette raison, nous exclurons, a priori, la pratique esthétisée et artistique du graffiti que représente l'art urbain et nous nous restreindrons au graffiti verbal.

\section{DÉFINITION DU PHÉNOMÈNE}

\section{Superposition et marginalité}

Le terme graffiti est problématique, car il relève du langage commun et non d'une terminologie linguistique. Il convient alors de proposer une définition terminologique qui n'entre pas en contradiction avec le sens courant. Le graffiti est une inscription qui répond à différentes propriétés. Il s'agit d'une inscription (1) SUPERPOSÉE et (2) MARGINALE. Ces deux propriétés définissent la nature du criterium du graffiti : à savoir son caractère extrinsèque.

${ }^{1}$ Il y a évidemment un nombre conséquent d'études, autres que sociolinguistiques ou sémiotiques. Nous pourrions ainsi citer des études relevant du domaine des sciences historiques et sociales, qui étudient le graffiti comme document ou comme indice; nous pourrions citer le domaine de la géographie, exemplifié par Ollive (2006), sur la spatialité du graffiti - notion analogue à celle d'urbanité. Nous nous sommes cependant restreint aux études qui concernaient notre approche. 
(1) Une inscription respecte la propriété dite de SUPERPOSITION si elle est extrinsèque à son support quant à la matérialité. En d'autres termes, si le support n'a pas été conçu pour y recevoir une inscription, le graffiti est en inadéquation avec ledit support. Une inscription sur un mur sera une inscription superposée. Une inscription sur les pages d'un livre, autre que les mots imprimés lors de sa conception, sera également une inscription superposée.

(2) Une inscription respecte la propriété dite de MARGINALITÉ si elle est extrinsèque à son support quant à sa situation au sein d'un système de normes restreint à l'usage de l'objet. La marginalité est un écart entre l'usage réel et l'usage supposé à l'objet lors de sa conception. Autrement dit, la MARGINALITÉ est un méta-niveau de la SUPERPOSITION, elle concerne le système de normes induit par la matérialité du support.

Remarquons que la MARGINALITÉ et la SUPERPOSITION, bien qu'étant des propriétés fondamentalement différentes, sont intimement liées et peuvent être confondues ou se superposer. La matérialité d'un objet est liée à la conception que l'on se fait de son usage, et l'usage d'un objet est lié à sa matérialité. Enfin, l'usage dans l'absolu est différent de l'usage réel, dépendant de restrictions sociales. Marginalité et superposition entrent dans une dialectique qui, d'un pur point de vue théorique, semble complexe et paraît neutraliser l'opposition. Néanmoins, cette opposition s'avère indispensable en pratique. Différencier ces deux propriétés est nécessaire, partant qu'elles doivent être présentes conjointement pour former un graffiti.

Procédons à l'aide de trois exemples concrets illustrant les différentes combinaisons et cas possibles :

(a) Soit une inscription sur un mur. L'inscription sera superposée, car elle est extrinsèque à la matérialité du mur; elle sera marginale, car le système de normes réglementant l'usage du mur ne permet a priori aucune inscription. Cependant, une autorité socialement compétente peut autoriser des inscriptions sur un mur, décrétant de facto la non-marginalité de l'inscription. Ainsi, si une municipalité demande à un graffeur de décorer un mur, l'inscription n'est, a priori, ni marginale ni superposée ${ }^{1}$.

(b) Soit un cahier de notes conçu pour recevoir l'écriture du professeur : un élève qui y apporte une inscription à l'insu du professeur réalisera une inscription marginale, puisque le système de normes quant à l'usage de l'objet interdit qu'un élève y écrive sans autorisation. Toutefois, il ne s'agira pas d'une inscription superposée, puisque la matérialité de l'objet implique que

1 Nous sommes obligé, dans un premier temps, de prétendre à une définition objective d'un objet qui ne peut être appréhendé que par la subjectivité du locuteur. Aussi, la non-superposition de l'écriture est éminemment subjective. 
l'on y écrive. Si, munis d'une craie, nous écrivons sur un tableau noir, l'inscription ne sera pas superposée puisque sa matérialité demande à ce que l'on y écrive - en adéquation avec son usage. Néanmoins, si nous écrivons dessus avec un marqueur indélébile, l'inscription sera superposée et marginale. Considérons à présent un élève qui écrirait sur un tableau noir disposé dans une salle de classe, sans autorisation, mais muni d'une craie. L'inscription sera marginale, eu égard aux prescriptions additionnées aux normes de base de l'usage. La disposition du tableau noir rend indu le comportement de l'élève - nous sommes à un degré supérieur de normalisation ${ }^{1}$.

(c) Si nous prenons un livre imprimé, dans sa finitude les caractères imprimés sur le papier ne sont pas superposés puisque la matérialité de l'objet livre a des caractères imprimés sur ses pages. Le produit fini possède les caractères imprimés, donc ils ne sont pas superposés. Par contre, annoter le livre laissera des inscriptions superposées. Par ailleurs, définir le statut de marginalité ou de non-marginalité de ces annotations demeure relativement complexe. Si les annotations sont personnelles, c'est-à-dire s'il s'agit des annotations du possesseur légitime du livre, elles ne sont vraisemblablement pas marginales, puisqu'elles peuvent être attendues, compte tenu de l'usage qui est fréquemment fait d'un livre - bien que l'usage en question ne soit pas le système d'usage prévu, contrairement à un livre d'exercice qui disposerait de lignes à cette fin. À l'instar du tableau noir, le livre a plusieurs degrés de normalisation. Le premier degré relevant du concepteur, et le second relevant du propriétaire et de sa propre perception de l'usage normal ${ }^{2}$ d'un livre.

Il apparaît toutefois que les propriétés de SUPERPOSITION et, particulièrement, de MARGINALITÉ, bien que congrues et opérantes, ne peuvent suffire à la description du graffiti. Remarquons d'ailleurs que cette opposition recouvre l'opposition éminemment linguistique, entre système et usage - d'où s'opérerait un écart. Il s'agit donc bien d'une opposition de méthode qui nous permet de définir une typologie pertinente et économique et non d'une opposition absolue. Ainsi à l'instar de l'opposition linguistique entre

${ }^{1}$ En termes de pure matérialité, nous pouvons considérer une inscription à la craie comme superposée. Toutefois, cette superposition est à un degré inférieur, pourrionsnous dire, d'une superposition inhérente à une inscription au marqueur indélébile, qui ne pourrait être effacé puisque la nature physique du support ne permet pas que soit effacé le marqueur - de facto écrire au marqueur est marginal tandis qu'écrire à la craie ne l'est que si une interdiction est proférée dans le lieu où se situe le tableau noir, il s'agit donc d'une interdiction liée à un système de normes moins général, localisable, en quelque sorte.

${ }^{2}$ L'emploi est considéré comme normal en fonction de la perception de l'allocutaire des normes d'usage - l'emploi qui doit être fait - et des normes statistiques d'usage l'emploi qui est fréquemment fait. 
système et usage, l'opposition entre usage réel et usage supposé au sein d'un système de normes doit être nuancée : il faut se garder d'hypostasier l'écart opéré entre le système de normes - qui est virtualité d'usage - et l'usage réel.

Soit un observateur omniscient - c'est-à-dire ayant une connaissance séparée et absolue du système et de l'usage -, il sera en mesure d'évaluer objectivement cet écart, tandis qu'un observateur empirique ne peut en avoir qu'une évaluation subjective - un jugement épilinguistique - en lien avec sa représentation singulière de l'usage supposé et du système qui l'encadre. Ajoutons encore que le système de normes n'est pas une donnée a priori ou reconstruite. Le système de normes est conçu par les acteurs qui en ont la légitimité. Autrement dit, parce qu'il ne permet pas de s'affranchir de la réalité de la perception de l'allocutaire, le critère de marginalité, bien que discriminant, n'est pas dirimant. L'écart n'est qu'une évaluation subjective reposant sur un système de normes légitimant ou non l'usage, et sur le degré de connaissance de ce système.

Par conséquent, la marginalité d'une inscription est un concept fondamental basé sur le ressenti d'un récepteur - à l'instar de la sensation d'écart. L'écart linguistique et l'écart que nous définissons ici apparaissent être un même phénomène réalisé à deux niveaux différents : la perception d'un locuteur eu égard à une représentation d'un système tantôt (intra-)linguistique et tantôt extra-linguistique. En effet, si l'énoncé est la résultante de la phrase et du contexte (Ducrot, 1984), nous pouvons considérer que l'écart (linguistique) provient de la distance prise entre l'énoncé et le système quant à la phrase, tandis que la marginalité est la distance prise entre l'énoncé et le système quant au contexte. Les implications de ce postulat sont (1) la non-dualité entre système et usage et (2) la non-dualité entre contexte et énonciation, sur laquelle nous reviendrons. De cette propriété procèdent la perception du sujet et l'aspect sociologique.

En effet, parce qu'une inscription est marginale elle sera perçue comme anormale et socialement marquée - et non l'inverse. Les propriétés de superposition et de marginalité, en plus d'être liées, sont dépendantes de la perception des individus. Alors qu'il ne s'agit pas d'un graffiti, l'inscription marginale sur le tableau noir ou sur le cahier de notations sera perçue tantôt comme une marque d'irrespect, tantôt comme un comportement anodin; tandis que pour certains, il n'est pas acceptable d'annoter un roman. Ils pourraient, dès lors, considérer l'annotation comme une inscription marginale. Nos deux propriétés rentrent donc dans une dialectique dynamique qui intègre dans une définition objective le rapport à la subjectivité du récepteur qui lit les graffitis - et qui les percevra comme tels en fonction du rapport conjoint des propriétés de marginalité et de superposition. Nous pouvons alors définir le graffiti: il s'agit d'un énoncé réalisé en manière d'inscription marginale superposée pouvant être pictographique ou iconographique, dont la perception 
et la reconnaissance dépendent de la subjectivité de l'allocutaire, ainsi que de sa perception du système de normes qui encadre l'usage du support sur lequel est inscrit l'énoncé. Cette définition rend compte du double aspect intriqué du phénomène : aspect matériel (nature physique du support et de l'écriture) et immatériel, voire logiciel (relation entre le support et le système de normes, perception du système).

\section{TYPOLOGIE TRIADIQUE}

Suite à nos observations, guidées par l'appareil théorique préalablement exposé, nous avons défini un corpus réduit contenant 250 graffitis relevés dans les toilettes pour hommes des bâtiments de la faculté de Philosophie et Lettres de l'Université de Liège. Sur la base de ce corpus, nous avons établi une typologie fondée sur un critère corrélé aux propriétés sus-développées : la relation locuteur-allocutaire-contexte (le lieu, le support, etc.). Notre typologie comprend les types suivants :

(1) Environnemental, c'est-à-dire lié à l'environnement matériel du support lui-même. Autrement dit, le graffiti rentre dans une dynamique interactive avec le support. En effet, ce type de graffiti détourne ou répond à un élément de l'environnement. L'ILLUSTRATION I comporte ici deux graffitis. L'un indiquant à l'aide d'une flèche que le sèche-main est hors service, l'autre répondant au premier. Le second exemple (ILLUSTRATION II) est intéressant quant à son contenu et au rapport qu'il entretient avec la situation d'énonciation. Si le précédent graffiti répondait effectivement à un élément de l'environnement, nous ne pouvions cependant y voir une dynamique complète entre les trois arguments de la triade. Ici, alors qu'il y a une seule énonciation, le nombre d'énoncés sera, en quelque sorte, indénombrable. En effet, le graffiti seul ne fait pas sens, le miroir participe donc, non pas tant de son interprétation, mais de son signifiant. Nous avons une unité sémiotique complexe composée d'un élément verbal et d'un élément non verbal. Ces graffitis environnementaux représentent $2,4 \%$ du corpus.

(2) Événementiel, c'est-à-dire lié au contexte temporel et à la temporalité d'un événement. Il s'agit de graffitis dont le référent ne peut se réaliser que par sa propre énonciation. Ce type de graffitis, s'apparentant à un acte performatif, consiste généralement en des invitations, souvent d'ordre sexuel. Ils représentent 7,2\% du corpus (ILLUSTRATION III)

(3) Dialogal, c'est-à-dire répondant à un autre graffiti, soit $39,2 \%$ du corpus (ILLUSTRATION IV et V).

(4) Ex nihilo, c'est-à-dire dépourvu de tout lien avec l'environnement. Ces graffitis forment le prototype d'une énonciation spontanée, soit $50,8 \%$ du corpus. Nous pouvons distinguer au sein des graffitis ex nihilo des graffitis 
mettant en scène le locuteur. Ces graffitis spectacularisants représentent $32 \%$ de cette catégorie, soit $16,4 \%$ du corpus. Nous pouvons les répertorier dans trois catégories, selon qu'ils spectacularisent :

(4.1) L'individu (ad hominem), qui fait le spectacle d'une individualité, soit $6,4 \%$ du corpus (ILLUSTRATION VI).

(4.2) La langue, qui fait le spectacle d'un emploi (usage du cyrillique, de l'anglais, d'un alphabet codé, etc.), soit 8,8\% du corpus (ILLUSTRATION VII).

(4.3) La personne (ad personam), qui fait le spectacle d'une situation personnelle (déclaration d'amour, etc.) soit 1,6\% du corpus (ILLUSTRATION VIII).

Chacune de ces catégories se définit par la relation entretenue dans la triade locuteur-allocutaire-contexte. Par opposition aux graffitis ex nihilo, nous pourrions définir les première et troisième catégories comme ex materia: le substrat présent étant tantôt un élément de l'environnement, tantôt un graffiti. Ils répondent à cet élément. La seconde catégorie est un cas particulier, que nous aborderons plus en profondeur, où le locuteur adresse une invitation, dans un lieu qui lui semble adéquat, pour un événement dont la condition de réalisation est la réception et l'exécution du message par un allocutaire. Nos graffitis ex nihilo spectacularisants cherchent à mettre en scène le locuteur aux yeux d'un allocutaire, particulier ou non. Enfin, les graffitis ex nihilo restants semblent être un mystère. Il est difficile d'établir une relation et d'en comprendre l'intention. À cela s'ajoute la difficulté suivante : il est possible de considérer les graffitis comme étant des éléments de l'environnement, malgré la possible contradiction avec la notion de superposition et de marginalité. Autrement dit, si nous considérons l'exemple suivant : le graffiti P.D détourné en P.DG. Nous pouvons considérer le $G$ ajouté comme une réponse ou comme un détournement - détournement alors d'un élément de l'environnement, et ranger notre graffiti tantôt dans la première, tantôt dans la troisième catégorie.

Afin d'affiner notre typologie, nous pouvons proposer un autre mode de catégorisation constitué de trois catégories auxiliaires et transversales, en rapport cette fois avec le contenu, qui regroupent des graffitis présents dans différentes catégories :

(5) Les graffitis outrageants qui représentent $24 \%$ du corpus (ILLUSTRATION IX).

(6) Les graffitis d'opinion qui relèvent soit de la spectacularisation de cette dernière, soit $\mathrm{du}$ contexte dialogal, représentent $17,6 \% \mathrm{du}$ corpus (ILLUSTRATION X). 
(7) Les graffitis métalinguistiques, qui sont liés au contexte d'énonciation strict et qui répondent à la forme et à la langue, soit $6.8 \%$ du corpus. Nous pouvons ajouter à cette catégorie les détournements langagiers (ILLUSTRATION $\mathrm{XI})$.

En opérant un recoupement entre ces deux modes de typologie, nous pouvons constater que $41 \%$ des graffitis outrageants sont ex nihilo. En d'autres termes, ces graffitis ne consistent pas uniquement en une réponse vulgaire, ou en un emportement discursif. Il s'agit d'insultes arbitraires obéissant à une forme de pulsion psycholinguistique, que nous étudierons au point 3.3. La cinquième catégorie regroupe les graffitis concernant une opinion politique, religieuse, sociale, etc. Il s'agit majoritairement de graffitis ex nihilo à vocation spectacularisante. Le locuteur écrit une opinion, de façon généralement synthétique et remarquable. Nous retrouvons ainsi des exemples de sigles euro barrés, Palestine écrit au marqueur, etc. À ces graffitis s'ajoutent des énoncés plus conséquents, moins synthétiques, reposant sur une vision stéréotypée, voire complotiste du monde politique: allusions à des complots franc-maçonniques, juifs, américains, etc. Un cas singulier toutefois: une opinion dépréciative visant les baptisés et la tradition universitaire du baptême ${ }^{1}$ (voir ILLUSTRATION XXI). À ces opinions répondent des graffitis d'ordre dialogal.

Enfin un dernier contenu se détache des autres par sa récurrence et sa transversalité. Transversal, car il peut s'articuler avec n'importe quel graffiti, quel que soit son contenu. Il s'agit des graffitis à contenu métalinguistique, qui sont tantôt des corrections orthographiques et des détournements, tantôt des énonciations stricto sensu. Dans le cas d'une correction ou d'un détournement, le locuteur ajoute un accord ou une lettre pour changer le lexème (le cas de $P . D G$ ). Évidemment, une simple correction est souvent accompagnée d'un propos justificatif. Le propos métalinguistique est généralement une technique de détournement argumentatif telle que la mutatio controversice. Si, parfois, il est utilisé comme élément rhétorique, il l'est bien plus souvent par moquerie. La présence de ces nombreux propos métalinguistiques témoigne par ailleurs du rapport complexe et insécurisé à l'orthographe.

${ }^{1}$ Le graffiti permet notamment d'exprimer un contenu illégitime ou polémique. Le cas du baptême est un exemple prégnant. Le baptême estudiantin en Belgique, auquel peu d'études sont consacrées, peut être défini comme un rite de passage folklorique. Différents codes sémiotiques marquent ce rite afin de différencier les baptisés des non baptisés. La pratique du baptême, par sa nature de rite de passage, sa similarité avec le bizutage et les discours discordants sur le sujet, est porteuse de nombreux stéréotypes et, par conséquent, voit les discours en question se cristalliser en un point de polémique. En l'occurrence, le graffiti permet à son auteur l'expression d'une opinion dépréciative, en direction d'étudiants potentiellement baptisés, déclenchant un débat par graffitis interposés. 


\section{CANAl ET SITUATION}

Il nous apparait que la typologie proposée est n'est pas suffisante d'un point de vue théorique. Certes pertinente empiriquement, car apte à distinguer et caractériser plusieurs types de graffitis qui divergent effectivement dans leur rapport à la situation d'énonciation, notre typologie repose néanmoins sur des critères théoriques trop peu homogénéisés : contenu, contexte, support, allocutaire, locuteur et intention - notion problématique. À la complexité de notre typologie s'ajoute celle de la définition du phénomène. Il est nécessaire, si nous désirons poursuivre son étude, d'homogénéiser les propriétés du phénomène selon un critère précis en adéquation avec sa définition et avec la typologie, construite pour y reconnaître différents individus.

Ce critère idoine est le concept de canal qui, repris à Jakobson, est depuis théorisé en linguistique par de nombreux auteurs (voir Gadet, 2007 ; Wüest, 2009 ; Lebray, 2012 ; Uygur, 2013), afin de rendre compte d'une catégorie de variations opérant entre l'écrit et l'oral. En augmentant l'extension définitionnelle de la notion de canal, par l'intégration de la notion de support ${ }^{1}$, nous pouvons synthétiser les éléments hétéroclites qui servent notre analyse. Le canal implique la nature matérielle de l'énoncé, les conditions d'usage, un locuteur et un allocutaire, un contexte et une situation contraignant ledit usage, une prédétermination du contenu et un conditionnement de la forme. Le canal subsume la somme des variables de l'énonciation et la somme des variations qui distinguent différents types d'énoncés. Le graffiti est un énoncé singulier parce qu'il procède d'un canal singulier. Définir son canal - c'est-à-dire le médium permettant l'énonciation - et en établir les propriétés, constitue une condition sine qua non à son étude. Les propriétés de superposition et de marginalité sont inhérentes à la matérialité du canal, à la nature du support physique qui l'actualise et au système normatif qui en encadre la gestion. Outre la matérialité et les variations qui lui sont inhérentes, le canal impose une variation plus abstraite, qui n'est plus de l'ordre du support mais de la médiation énonciative. Il s'agit de la prédétermination du contenu et du conditionnement de la forme.

Pour étudier les variations entre énoncés, propres aux canaux qu'ils utilisent ou à l'usage différencié d'un même canal, il nous semble à propos d'utiliser le concept corolaire de variation diamésique. Bien que de nature écrite, notre phénomène semble s'éloigner du parangon de l'énoncé écrit. Il est pertinent de considérer le graffiti comme un énoncé singulier se situant à proximité du pôle prototypique écrit. Ce pôle formerait, avec le pôle prototypique oral, les deux extrémités d'un spectre sur lequel nous pouvons

\footnotetext{
${ }^{1}$ Selon nous, nous ne pouvons pas réduire la notion de canal au canal oral et écrit. Le canal écrit se décline en une multitude de canaux, selon le support et les modalités qui lui sont propres : par exemple, le support numérique (Pignier, 2010).
} 
situer la pratique que nous étudions. La variation diamésique est intimement liée aux notions de temporalité et d'espace comme le montrent les notions d'immédiat communicatif et de distance communicative, développées par Koch et Oesterreicher dans leur tentative de définition (Wüest, 2009).

En effet, l'énonciation orale s'étend dans le temps et entretient un rapport singulier à la temporalité qui l'encadre et à la position du sujet dans cette temporalité. Nous retrouvons ainsi les phénomènes d'accident ou de variation selon que l'énonciation est actualisée dans un canal strictement oral ou dans des canaux non acoustiques (langage des signes par exemple). Les variations diamésiques, entre les différents canaux, se fondent alors sur des critères variés. Aux propriétés matérielles et physiques (acoustique, visuel, ou tactile pour le braille) ou cognitives (écriture cursive ou clavier), aux modalités propres au support (voir Pignier 2010), s'ajoutent les propriétés situationnelles (conversation en différé, etc.), ou épiphénoménales (présence ou non d'indices mimogestuels). Ces variations touchent différents niveaux du langage (sémiotique, discursif, phrastique, morphologique, graphique) et se combinent à d'autres variations linguistiques: diaphasiques (l'usage épistolaire et l'écriture d'un mail demandent un certain registre ${ }^{1}$ ), diastratiques, etc. Les énoncés graffitis vont donc présenter certaines propriétés formelles en fonction des contraintes et modalités du canal ou du besoin de pallier une propriété dont il ne dispose - des stratégies pour donner à l'écrit quelque chose de l'oral.

Ces variations permettent de définir diverses énonciations hétéroclites. Songeons aux pratiques de correction, de soulignement ou de détournement que nous rencontrons dans les graffitis. La nature de ces énoncés est liée au canal dans sa matérialité et dans ses modalités (possibilité du palimpseste, etc.). D'aucuns pourraient néanmoins objecter que ces éléments disparates ne relèvent pas de l'énonciation et ne sont pas des énoncés. Il s'agit pourtant d'énoncés marqués par des variations diamésiques et s'éloignant, en conséquence, du prototype écrit. À cela s'ajoutent les spécificités diamésiques propres à la nécessité d'articuler les énoncés dans l'espace qu'est un support en deux dimensions ne disposant d'aucune règle d'organisation: la

\footnotetext{
${ }^{1}$ Gadet constate déjà la perméabilité entre diamésie et diaphasie (2007). Il convient toutefois de les différencier et d'analyser leur combinaison. L'écriture d'une lettre, à l'instar d'un mail, oblige la sélection d'un registre particulier, associé à la pratique épistolaire. Aussi, bien qu'étant situées à deux points différents du spectre diamésique, les deux pratiques partagent un style semblable. Remarquons toutefois que le registre associé à la pratique du mail n'est pas encore totalement soumis à une norme formalisée. Le manque de normes quant au registre est à l'origine de variations possibles entre la perception du ton par l'allocutaire et le locuteur. A contrario, le clavardage (discussion instantanée en ligne ou tchat), se différencie du mail au niveau diaphasique, alors qu'ils partagent la même position sur le spectre diamésique.
} 
marginalité de l'énoncé implique en effet l'absence de normes et de prescriptions organisatrices. Le graffiti doit alors s'insérer dans l'espace et, à l'aide d'un balisage, être circonscrit. Nous retrouvons différents moyens d'effectuer ce balisage - communs ou non à d'autres pratiques de l'écrit : ponctuation, majuscule, encadrage, typographie, coloriage, etc. L'énoncé doit ensuite s'articuler avec les autres énoncés insérés dans l'espace - que ce soit pour y répondre ou marquer l'indépendance par rapport à ceux-là. Nous retrouvons ainsi l'usage de flèches, d'encadrements, etc. (voir ILLUSTRATION XII, XIII et XIV).

Le canal implique deux autres propriétés : le palimpseste et l'anachronie. Effectivement, la nature spatiale du support (qui actualise physiquement le canal) permet de superposer plusieurs énoncés. Cela implique la disparition des énoncés précédents au profit de nouveaux, ou une pratique du détournement et de la parodie par la modification d'un énoncé précédent dont résultent les phénomènes d'énoncés non strictement identifiables. L'anachronie est l'incapacité d'identifier dans le temps l'énonciation. Il n'y a pas de synchronie entre l'émission et la réception, entre l'énonciation et la lecture - ce qui est en adéquation avec les paramètres développés par Koch et Oesterreicher afin de mesurer la distance communicative ${ }^{1}$ (Wüest, 2009). Cela implique l'anonymat du locuteur. De cette incapacité découle le besoin du locuteur de dater ces épigraphes et de les signer pour lever l'anonymat - par un pseudonyme ou un indice.

\section{CAUSALITÉS DU PHÉNOMÈNE}

\section{Activité de l'énonciateur}

Ces deux propriétés ont des répercussions formelles remarquables. Nous pourrions répertorier, pour chaque type de graffitis, les actualisateurs présents : pronom de la première, de la seconde ou de la troisième personne ou nom propre. Nous pouvons ajouter à ces actualisateurs les pseudonymes, la datation et l'articulation d'un graffiti avec un autre, pour lui répondre, au moyen de flèches ou d'autres indices. Cependant, ces éléments formels ne font sens que si nous les étudions selon le conditionnement qu'implique le canal, ou plutôt qu'implique l'usage du canal par le locuteur dans une situation donnée. Il nous faut expliquer le motif du locuteur dans le choix d'un tel canal, c'est-à-dire établir quelle est la situation du locuteur pour qu'un tel canal énonciatif s'impose. De la situation d'énonciation découle l'usage d'un canal approprié mais, inversement, le choix du canal modifie en conséquence

\footnotetext{
${ }^{1}$ Koch et Oesterreicher définissent un continuum entre deux pôles - l'immédiat communicatif et la distance communicative - caractérisés par une dizaine de paramètres.
} 
la situation d'énonciation. Le canal est au cœur de notre problématique puisqu'il est la cause des caractéristiques de l'énoncé et la conséquence d'une médiation entre le locuteur et le monde dans lequel il s'installe par son énonciation.

Le canal ne peut s'expliquer que par la situation de l'énonciateur dans le monde. Nous devons donc prendre en compte : l'activité de l'énonciateur, le lieu où il énonce, et le temps dans lequel il énonce. Pour mettre au jour son activité, il convient de procéder par une distinction nouvelle : une énonciation peut être préméditée ou spontanée.

(1) L'énonciation est préméditée si le locuteur prévoit son énonciation en fonction du contenu et du lieu. Dans le cas du graffiti, le locuteur cherche un lieu pour y écrire, souvent particulièrement visible ou spectaculaire. Il s'agit donc toujours d'une spectacularisation du graffiti qui consiste soit en une signature stylisée, en l'écriture d'un blase, soit en un message politisé - de type pochoir, etc.

(2) L'énonciation est spontanée si le locuteur n'a pas étudié préalablement son énonciation en fonction du contenu et du lieu. Bien que l'énonciation des graffitis soit favorisée et conditionnée par le lieu - en l'occurrence les toilettes -, elle n'a pas été anticipée par le locuteur. Ce dernier ne s'est pas rendu aux toilettes dans le but d'y réaliser un graffiti - bien qu'il puisse exister des exceptions.

Les graffitis qui constituent notre corpus sont a priori tous spontanés. Ceux-ci sont liés au repos, voire à la passivité de l'énonciateur. C'est lors d'un moment d'ennui ou d'oisiveté qu'il produit des graffitis et autres gribouillages. La passivité, particulièrement lors de l'écoute, est souvent associée à une production parasite - allant de simples gribouillages à des énonciations. Psycho-linguistiquement, le graffiti pourrait donc être lié à cette passivité, notamment lors de l'écoute - médecin à l'écoute de symptômes, élèves à l'écoute du professeur - ainsi qu'à l'oisiveté lors de la patience (aux toilettes, à un arrêt de bus, etc.). Notons toutefois que la frontière entre gribouillage et graffiti peut paraître ténue, bien qu'analogue à la frontière distinguant une activité linguistique et une activité graphique dépourvue de sens. Aussi, certaines études (Gombrich 1999, Andrade 2009, Schott 2011) se sont intéressées à l'activité parasitaire du gribouillage (doodling). S'il apparaît que l'étude d'Andrade donne au gribouillage une fonction que nous ne pouvons imputer aux graffitis, elle nous permet toutefois de remarquer la similitude du contexte de manifestation de ces deux activités. Elles apparaissent dans un moment de patience rendant possibles l'ennui et la rêverie (Andrade 2009). Nous nous contenterons donc, dans un premier temps, de considérer que le contexte de patience et de passivité est le déclencheur d'une activité linguistique spontanée et, peu ou prou, parasitaire. 
Si ce contexte est un déclencheur, il ne suffit pas à expliquer la présence des graffitis. À cette considération, nous devons ajouter des déclencheurs subsidiaires, ou plutôt des incitations. Une incitation remarquable est la présence même de graffitis. Ils incitent premièrement à une mise en discours. Cette incitation est presque de l'ordre de l'énonciation. En effet, si un graffiti prend la forme d'une question, le locuteur en position de passivité-patience aura une forte tendance à y répondre. Nous avons ici un phénomène purement linguistique. Ils incitent deuxièmement par désinhibition. La présence de graffitis étant souvent considérée comme une détérioration, le lieu où le locuteur est en état de patience sera perçu par ce dernier comme détérioré et donc comme pouvant l'être encore plus - que ce soit par de nouveaux graffitis, avec ou sans rapport avec ceux déjà présents, ou par des actes d'autres types. Ce phénomène rentre dans le cadre de l'hypothèse dite de « la vitre brisée », selon laquelle une détérioration en appelle une autre (Wilson, Kelling, 1982).

Les toilettes sont évidemment concernées par cette idée de patience. De plus, ce lieu a un statut anthropologique particulier participant de l'écriture du graffiti. Le lieu fonctionne comme un déclencheur rendant possible et pertinente l'énonciation - la justifiant. L'énonciation est, en quelque sorte, l'actualisation de la position du locuteur dans un lieu donné, dans une situation d'énonciation donnée. La situation d'énonciation ne permet pas seulement d'appréhender l'extension d'une expression - qui est secondaire mais la valeur sémiotique de l'acte de langage lui-même. L'énoncé ne fait sens qu'au regard de la situation d'énonciation. Autrement dit, le contenu de l'énoncé importe peu, mais le fait d'énoncer est signifiant, compte tenu de la situation. Dès lors, pour analyser la situation d'énonciation nous devons analyser la situation selon deux éléments fondamentaux : l'espace, c'est-à-dire le lieu, et le temps.

\section{Situation de l'énonciateur : la question de l'espace et du temps}

L'espace dans lequel nous avons étudié le graffiti est un lieu particulier : les toilettes pour hommes. Si nous nous en tenons à la définition de Marc Augé, la toilette est le parangon du non-lieu: ni identitaire ni relationnel (Augé 1992). La toilette est un lieu de passage fréquenté par un nombre conséquent d'individus inconnus les uns des autres, dont l'usage est lié à une activité commune à tous les individus en question et socialement connotée : la réalisation d'un besoin biologique. Non-lieux par excellence, les toilettes semblent partager certaines propriétés de l'hétérotopie, telle que définie par Foucault: «[...] des lieux qui sont dessinés dans l'institution même de la société, et qui sont des sortes [...] d'utopies effectivement réalisées dans lesquelles les emplacements réels, tous les autres emplacements réels que l'on 
peut trouver à l'intérieur de la culture sont à la fois représentés, contestés et inversés, des sortes de lieux qui sont hors de tous les lieux, bien que pourtant ils soient effectivement localisables » (Foucault, 1984, 4).

Il peut sembler dépourvu de pertinence d'attribuer ces caractéristiques aux toilettes. Toutefois, il s'agit bien de lieux réels, dessinés dans l'institution même de la société au point qu'ils soient pourvus d'un corps social et d'une économie propres, comme l'indique la présence d'un métier nommé et reconnu dans l'institution sociale (désigné par le $\mathrm{ROME}^{1}$ comme «gardien de toilette (K2501) »). Ce lieu est hors de tout autre dès lors qu'il circonscrit un espace dédié à une activité considérée comme taboue, dont procède l'humour scatologique. De plus, la présence des graffitis participe de ce lieu. En effet, ils réalisent et incarnent les représentations, contestations et inversions dont parle Foucault, en témoignent le nombre de graffitis outrageants, spectacularisant une opinion rejetée, ou invitant à l'assouvissement sexuel. Les toilettes publiques permettent une situation d'énonciation modulée selon un mundus inversus moderne. La combinaison de la situation de patience du locuteur, du lieu hétérotopique et non identitaire («non-lieu»), des énoncés déjà présents fonctionnant comme déclencheurs énonciatifs et comme désinhibiteurs normatifs, amène le locuteur à énoncer un contenu carnavalesque - et en particulier dans une université.

En outre, le temps participe autant de l'énonciation particulière qu'est le graffiti que le lieu. Nous avons insisté sur l'état de patience, aussi nous faut-il apporter un éclaircissement à l'effet de l'attente sur le besoin de produire un énoncé. En effet, l'énoncé graffiti nous semble être, avant tout, la conséquence d'un besoin essentiel d'énoncer. Similaire au besoin d'exprimer vocalement un monologue intérieur, le graffiti semble provenir de la nécessité de l'individu solitaire de produire de la voix. Dans cet instant de patience où le locuteur se retrouve seul, passif, parfois confronté à des énoncés, et dans un espace propice, nous pouvons formuler l'hypothèse qu'il y a un besoin de produire une énonciation, de s'installer dans le temps qui s'écoule comme sujet présent et actif. Nous pouvons rapprocher la position passive du locuteur dans cette action à la notion de "position dépressive dans la production discours " (Barbéris, 2008, 106).

Ainsi, Barbéris développe que « le sujet qui vit le temps en descendance le voit 'venir à lui', avec le cortège d'événements qui 'lui arrivent' : il ne peut qu'en éprouver la pesée, dans une vision dépressive du temps » (Barbéris, 2008, 106). Nous pouvons prolonger cette conception et, selon l'idée pascalienne, considérer que la passivité discursive dans le temps, qu'est ce

\footnotetext{
${ }^{1}$ Répertoire Opérationnel des Métiers et des Emplois, disponible sur le site internet du Pôle Emploi.
} 
moment de solitude et de passivité dans un espace clos, est angoissante. Le discours intérieur ne peut se retenir d'éclater et d'imposer cette " présence au monde que l'acte d'énonciation rend seul possible», à savoir l'extériorisation du monologue intérieur - qui est déjà discours (Barbéris, 2008, 96).

En cet instant du dire, cette instance qui permet au je de se poser s'actualise, par la temporalité, la réalité du lieu. Le hic et le nunc deviennent hic et nunc intrinsèques à l'énonciation, il $\mathrm{y}$ a articulation du temps et de l'espace du sujet qui en forment le présent. Cette situation très particulière est à l'origine d'une pulsion. Procèdent de cet acte une série d'autres actes qui sont des actes de transformation du monde (Barbéris, 2008, 109). L'acte de parole a ici une action sur le monde qui dépasse l'ordre linguistique et prend une dimension sociale, voire anthropologique. Il transforme concrètement le lieu et le rend ensuite plus propice à l'acte de langage suivant - en pourvoyant l'espace d'un énoncé auquel il s'agira peut-être de répondre, et en participant à la consécration du lieu comme locus inversus. La situation n'est plus le cadre de l'énonciation, elle lui est consubstantielle. Le locuteur dans cette situation singulière sélectionne un canal tout aussi singulier pour réaliser, par l'acte de parole, sa médiation au monde.

Cette médiation a des propriétés formelles remarquables. Nous pouvons constater que $57 \%$ des graffitis sont dépourvus d'actualisateurs. Parmi ceuxci, la majorité est représentée par ceux d'opinion - se résumant effectivement souvent au nom d'un parti politique, un appel à l'insurrection - et des graffitis ex nihilo. Ils sont totalement spontanés et relèvent de la pulsion, se résumant en une phrase nominale, en une interjection. Lorsque nous trouvons des actualisateurs, il s'agit majoritairement de pronoms à la deuxième personne particulièrement présents dans les dialogaux. Ils permettent de répondre ou de prendre à partie. Mais cela reste des pronoms vides, dépourvus de référent réel, puisque s'actualisant dans un lieu non identitaire, où la communication est différée, voire nulle. Les autres actualisateurs sont relativement peu fréquents. En effet, $12.8 \%$ des graffitis sont pourvus d'actualisateurs à la première personne et $12.4 \%$ à la troisième personne. $11.6 \%$ des graffitis ont des noms propres. Le sujet ne s'instaure pas en relation avec le lieu. Il n'y a, par ailleurs, que peu de combinaisons d'actualisateurs (voir ILLUSTRATION XXVI).

$\mathrm{Si}$ nous raisonnons par type de graffitis, nous constatons que les événementiels ont, en toute logique, un grand nombre d'actualisateurs à la troisième personne, ceux-ci étant pour la plupart une invitation émanant d'un locuteur représenté par la troisième personne à laquelle il substitue son je. Le lieu, de par les qualités évoquées, est idéal pour ce type d'invitation. Nous l'avons dit, les graffitis fonctionnent selon un agencement propre au canal qui permet d'éviter les actualisateurs. Il s'agit souvent d'interjections, de motsphrases, qui ne nécessitent aucun ancrage du discours. De plus, la mise en 
discours des graffitis, la manière dont ils se répondent, suit un principe de juxtaposition épurée. Les noms propres, lorsqu'ils sont utilisés, le sont le plus souvent dans les spectacularisations ad personam et ad hominem.

Sont présents aussi des actualisateurs moins formels : l'usage de dates $(6 \%$ des graffitis) et de pseudonymes. Les graffitis événementiels sont ainsi toujours signés d'une adresse e-mail permettant de contacter la personne concernée. Ils comportent souvent une date, actualisant dans le temps l'invitation. Les dates se retrouvent dans certains graffitis dialogaux. Dans les quelques cas de débats relevés (plusieurs graffitis se répondant sur un sujet donné), les graffitis étaient datés, comme pour permettre de se situer temporellement par rapport aux autres réponses, compensant ainsi la communication différée. Les dates et les signatures permettent à l'énonciateur de ne pas s'effacer dans la masse des graffitis. Le sujet se positionne face aux autres par son énonciation qui, pour l'allocutaire suivant, s'effacera dans la totalité de tous les discours. L'espace et le temps sont les facteurs principaux de cet effacement. Le temps implique une anachronie et un anonymat, renforcés par le lieu qui est non identitaire. Cette situation temporelle exige irrémédiablement l'allocutaire à répondre, ne serait-ce qu'intérieurement, et à se positionner potentiellement en énonciateur - énonciateur qui n'attend aucune réponse d'aucun allocutaire.

\section{Le cas de la blasphémie : une réponse psycholinguistique?}

Pour compléter notre analyse des causalités, il faut s'intéresser au graffiti outrageant, évoqué dans la typologie. La notion d'outrage permet de considérer ces graffitis de façon objective, partant que l'outrage est le dépassement d'une limite instituée par un système de normes. Ainsi, bien que la perception de ce système soit subjective, puisque l'évaluation de l'outrage dépendra de l'allocutaire, la notion d'outrage reste objective puisqu'elle se réfère à un système extérieur à l'allocutaire. Pour définir cette notion d'outrage, nous avons sollicité la notion de blasphémie, introduite par Benveniste (1947, 254-257) alors qu'il cherchait à définir le juron. En nous inspirant de son travail, nous pouvons définir la blasphémie comme l'action conjointe d'un outrage et d'un affranchissement du tabou linguistique lié à l'outrage.

La blasphémie fonctionne grâce à la violation d'un tabou linguistique qui permet alors l'apaisement du locuteur au moyen d'une décharge émotionnelle. Nous pouvons identifier le but de l'énonciation (décharge émotionnelle) et son fonctionnement (outrage et tabou). La blasphémie dépasse par ailleurs de loin le tabou religieux. Celui-ci ne doit pas être perçu comme une puissance extrinsèque et transcendante : il est profondément lié à la société et change en conséquence. L'outrage n'est plus religieux, comme le définissait Benveniste 
dans son article, et le tabou linguistique n'est plus le nom de Dieu. Il nous faut ensuite différencier le juron de l'insulte. Si l'un comme l'autre partagent l'outrage-tabou et la décharge émotionnelle, le juron est distinct de l'insulte quant au destinataire. Dans le cas du juron, il n'y a aucun destinataire, car il est lié à une situation objective; dans le cas de l'insulte, il y a une adresse à un destinataire.

Nous pouvons par conséquent différencier trois types de blasphémie dans nos graffitis outrageants : l'insulte, le pseudo-outrage et la blasphémie stricto sensu. L'insulte consiste en un propos offensif et irrespectueux envers un destinataire (parfois nommé), et marqué sur l'axe diaphasique par le choix d'un registre vulgaire. Les propos insultants spectacularisant la polémique se retrouvent toujours dans les contextes dialogaux. Il s'agit de lexèmes visant à apostropher le précédent locuteur, connard étant l'occurrence la plus répandue. Nous pourrions formuler l'hypothèse d'un jeu, d'une contrainte stylistique, analogue aux principes de la joute verbale. Ces graffitis, bien que présents dans un contexte de dialogue, spectacularisent, en quelque sorte, la divergence d'opinions, la réponse, et, de facto, l'individualité du locuteur capable, dans l'instant de son énonciation, de prendre à partie la totalité des locuteurs précédents effacés en une seule entité (voir ILLUSTRATION XXVII)

Le pseudo-outrage consiste en un usage détourné, presque métaphorique, de l'insulte, afin de mettre en scène par l'outrage les invitations d'ordre sexuel, en spectacularisant la misogynie et la vulgarité sexuelle. À la différence de l'insulte, le pseudo-outrage n'est pas offensif (mais invitatif), il participe du jeu du graffiti événementiel. Les termes salope, pute fonctionnent comme des apostrophes représentant l'allocutaire, tandis que les verbes crus font référence à un lexique pornographique attendu dans ce type d'invitation, qui suit toujours le même canevas (voir illustration XXVIII). La blasphémie stricto sensu, quant à elle, est le parangon de l'outrage. Elle repose sur un processus différent : la rupture d'un anathème.

Avec l'évolution de la société, la blasphémie ne repose plus sur un outrage religieux, mais bien sur un outrage sociétal lié à différentes réalités prostitution, domaine biologique ou fécal, rapports sexuels, classe sociale, etc. - et sur le tabou linguistique du mot qui évoque ces réalités. Ainsi, l'exclamation Putain est bien une blasphémie : il y a outrage et tabou puisque le mot est marqué vulgairement - d'où l'importance de la vulgarité. Nos graffitis s'éloignent de l'insulte en ce qu'ils ne s'adressent à aucun allocutaire particulier, mais ne s'approchent pas pour autant du juron, censé être lié à une situation nécessitant cette interjection. Par ailleurs, ils peuvent s'approcher de l'insulte, dès lors que le lecteur le prend pour lu. Considérons les cas de Connard ou $P D$, il peut autant s'agir d'une blasphémie que d'une insulte s'adressant aux potentiels allocutaires. Le plaisir de la blasphémie provient d'ailleurs de cette réception potentielle par n'importe quel allocutaire. 
Autrement dit, ces graffitis flottent entre l'insulte pure et simple et la blasphémie. Ils ont néanmoins la caractéristique de n'être pourvus d'aucun actualisateur: pas de $t u$, ni de verbe, juste le mot, si possible le plus bref possible (ILLUSTRATION XV, XVI et XVII).

Le pivot linguistique de ces graffitis est le tabou. Ces graffitis appartiennent tous au type ex nihilo, qui ne comporte aucune marque permettant d'identifier l'énonciateur. Ils sont anonymes et sans destinataire réel - ce qui les différencie notamment de l'insulte. Cette nature en fait un vecteur logique pour que s'y expriment toutes les frustrations, fussent-elles linguistiques - expression du dialecte - ou politiques - expression de propos extrêmes en réaction à un silence qui peut sembler imposé. Pour cette raison, un nombre incalculable de propos politiques sont soit liés à des idéaux politiques marginaux, dont l'énonciation est sujette à critique, soit à des propos qui relèvent de certains fantasmes. Le graffiti est une façon de prendre la parole et de la rendre visible au plus grand nombre, en conservant anonymat et impunité. Le mur est canal et média donnant indistinctement la parole à qui l'investit. Aussi, certains graffitis d'opinion s'approchent, voire se confondent, avec le graffiti outrageant lorsque l'opinion est un tabou - parfois liée à un tabou linguistique. La rupture de l'anathème est donc le prototype du graffiti outrageant. Nous retrouvons ainsi un grand nombre de croix gammées dont la réalisation n'a d'autre intérêt que l'affranchissement lié à cette rupture. Rupture, aussi, de tabous onomastiques. Nous retrouvons, outre les croix gammées, des occurrences d'Hitler, Shoah, ou, plus étonnamment, une occurrence de Garaudy et Duprat. L'exemple le plus saillant est celui de Dutroux, nom extrêmement connoté en Belgique, qui a de multiples occurrences dans notre corpus et n'a d'autre intérêt que le plaisir de s'affranchir d'un tabou'.

${ }^{1}$ L'occurrence, certes unique, de Duprat et Garaudy est étonnante. Bien que leur énonciation respecte les propriétés du graffiti outrageant blasphémique - il s'agit de graffiti ex nihilo consistant uniquement en l'énoncé onomastique -, ces deux cas s'éloignent légèrement de la blasphémie. En effet, contrairement au cas Hitler, les noms de Garaudy et Duprat ne sont pas ancrés dans la mémoire collective. Ainsi, bien que leur énonciation évoque des propos idéologiquement marginaux, et par conséquent marqués d'un certain tabou, celle-ci est trop référentielle, c'est-à-dire liée à un contexte historique précis et non atemporel, pour être un véritable tabou. En outre, Dutroux est l'exact opposé du cas étonnant de Duprat et Garaudy. Derrière ce nom propre il n'y a aucune référence à une quelconque idéologie ou politique ne pouvant être exprimée publiquement. Il s'agit uniquement d'énoncer un nom associé à un traumatisme d'ordre historique: l'affaire criminelle Dutroux, en 1996, qui a marqué la mémoire collective belge. 


\section{CONCLUSION}

$\mathrm{Au}$ travers de cette analyse, nous avons cherché à identifier et à circonscrire l'application de multiples notions. S'il nous fallait synthétiser notre étude, nous pourrions établir le constat suivant : une énonciation est coextensive à son canal, et inversement. Le canal est consubstantiel à la situation d'énonciation. Une situation donnée implique l'usage d'un canal particulier qui modifie la situation elle-même. Le graffiti est particulier par le rapport qu'entretient l'énoncé avec sa situation. La situation énonciative du graffeur, à savoir la combinaison d'une temporalité descendante, d'une passivité et d'un lieu propice à la désinhibition, dont les éléments constituants sont potentiellement supports pour une inscription, provoque un besoin énonciatif dont le canal ne peut être que celui du graffiti. Le contenu est donc prédéterminé par le canal puisque le canal est, en l'occurrence, dans sa matérialité et son actualisation, la situation elle-même. La situation induit un énoncé qui ne peut se réaliser que par la transmutation de la situation-même en canal.

Le canal cristallise la situation d'énonciation, prédétermine le contenu, détermine la forme qui pallie l'anonymat et à l'anachronie, tout en portant les stigmates de la spontanéité de l'énonciation, réalise effectivement l'énonciation et la concrétise physiquement à travers ses propriétés matérielles, positionnant ainsi l'inscription dans le spectre diamésique et, subséquemment, comme une pratique localisable au sein d'un ensemble de normes. Ce canal permet le positionnement du sujet à travers la totalité sociale dans laquelle il se dissout ensuite. Le graffiti nous apparaît être un énoncé répondant au besoin du locuteur de se situer dans le temps, de se réapproprier l'espace, d'actualiser le monde qui l'entoure en l'énonçant et, par conséquent, en le modifiant.

\section{BIBLIOGRAPHIE}

ANDRADE J., 2009, "What Does Doodling Do », Applied Cognitive Psychology n ${ }^{\circ}$ 24, John Wiley \& Sons, Hoboken, 100-106.

AUGÉ M., 1992, Non-Lieux, introduction à une anthropologie de la surmodernité, Le Seuil, Paris, 150p.

BARBÉRIS J-M., 2008, «Instant du loquor, instant du dire, instance de discours : du temps au sujet », Cahiers de praxématique $\mathrm{n}^{\circ} 51$, Laboratoire Praxiling, Montpellier, 87-110.

BENVENISTE E., 1947, «Blasphémie et l'euphémie », Problèmes de linguistique générale II, Gallimard, Paris, 254-257.

BERTONCINI P., 2010, Le tag en corse: analyse d'une pratique clandestine, L'Harmattan, Paris, $320 \mathrm{p}$. 
BLOCH-RAYMOND A., 2002, « Les façades de la désobéissance. Tags, graffs et fresques murales ", Revue des sciences sociales, $\mathrm{n}^{\circ} 24$, Presses Universitaires de Strasbourg, Strasbourg, 92-99.

BOUDINET G., 2003, Pratiques tag. Vers la proposition d'une «transeculture », L'Harmattan, Paris, 208 p.

BUSQUETS S., FELLONEAU M-L., 2001, Tags et grafs : les jeunes à la conquête de la ville, L'Harmattan, Paris, $205 \mathrm{p}$.

CALO F., 2003, Le Monde du graff, L'Harmattan, Paris, 158 p.

Chorong Y., 2014, Graffiti et Street art: étude des discours historiographiques et de la critique esthétique d'une forme sociale de modernité visuelle, thèse de doctorat en Histoire de l'art, soutenue le 16-122014, disponible en ligne sur archives.ouvertes.fr. URL: tel.archivesouvertes.fr/tel-01168748/file/31785_YANG_2014_archivage.pdf (dernière consultation le 5/04/2016).

DÁVILA C. R., 2009, «Les images de la ville. Une approche à la sémiotique urbaine», Penser la ville - approches comparatives, Centre Universitaire de Khenchela, Khenchela, 237-247.

DERYCKE M., 2003, «Les graffitis bateliers : Empreintes, suspensions nomination », Langage et société, $\mathrm{n}^{\circ} 103$, Éditions de la Maison des sciences de l'homme, Paris, 79-115.

DUCROT O., 1984, Le dire et le dit, Éditions de Minuit, Paris, 237p.

FOUCAULT M., 1984, «Dits écrits, Des espaces autres (conférence au Cercle d'études architecturales, 14 mars 1967)», Architecture, Mouvement, Continuité $\mathrm{n}^{\circ} 5$, Groupe Moniteur, Paris, 46-49.

GADET F., 2007, La variation sociale en français, Ophrys, Paris, 186 p.

GOMBRICH EH., 1999, "Pleasures of boredom », The uses of images : studies in the social function of art and visual communication, Phaidon, Londres, 287-288.

LEBRAY T., 2012, Variation linguistique et enseignement de la norme : l'exemple de la phrase interrogative, mémoire disponible en ligne sur archives.ouvertes.fr. URL : http://dumas.ccsd.cnrs.fr/dumas00781754/document (dernière consultation le 5/04/2016), $73 \mathrm{p}$.

MENSCH N., 2013, «L'art transgressif du graffiti. Pratiques et contrôle social », Sciences Humaines Combinées $\mathrm{n}^{\circ} 14$, disponible en ligne. URL : http://revuesshs.u-bourgogne.fr/lisit491/document.php?id=1449 (dernière consultation le 5/04/2016).

OLLIVE A., 2006, Graffitis et graffiteurs dans la ville : pratiques spatiales des graffiteurs de Québec et marquage symbolique de l'espace urbain, mémoire disponible en ligne. URL : www.theses.ulaval.ca/2006/23966/23966.pdf (dernière consultation le 5/04/2016). 
OUARAS K., 2009, " Les graffitis de la ville d'Alger : Carrefour de langues, de signes et de discours. Les murs parlent... », Insaniyat $\mathrm{n}^{\circ} 44-45$, Centre de recherche en anthropologie sociale et culturelle, Oran, 159-174.

OUARAS K., 2015, «L'espace urbain algérois à l'épreuve de ses graffiti », L’Année du Maghreb n²12, CNRS Éditions, Paris, 157-179.

PIGNIER N., 2010, «Le modèle du livre : dans les générateurs de documents numériques », Protée n ${ }^{\circ} 8$, UQAC, Chicoutimi, 73-80.

SCHOTT GD., 2011, "Doodling and the default network of the brain », The Lancet Neurology n ${ }^{\circ} 378$, The Lancet, 1133-1134.

SPINELLI L., 2007, «Pichação e comunicação: um código sem regra », Logos 26, UERJ, Rio de Janeiro, 111-121.

UYGUR D., 2013, La variation diamésique/diaphasique des marqueurs discursifs «Alors » et " quoi », communication au colloque « La dia-variation en français actuel. Des corpus aux ouvrages de référence (dictionnaires/grammaires) » de l'Université de Sherbrooke, Québec, disponible en ligne. URL : http://hdl.handle.net/2078.1/144086 (dernière consultation le 5/04/2016).

WILSON J. ET KELLING G., 1982, «Broken Windows: The police and neighborhood safety », The Atlantic., republication en septembre 2007, disponible en ligne. URL: http://www.manhattaninstitute.org/pdf/_atlantic_monthly-broken_windows.pdf (dernière consultation le 5/04/2016).

WÜEST J. (2009), « La notion de diamésie est-elle nécessaire ? », Travaux de linguistique, ${ }^{\circ} 59$, De Boeck Supérieur, Louvain-la-Neuve, 47-162.

\section{ILLUSTRATIONS}




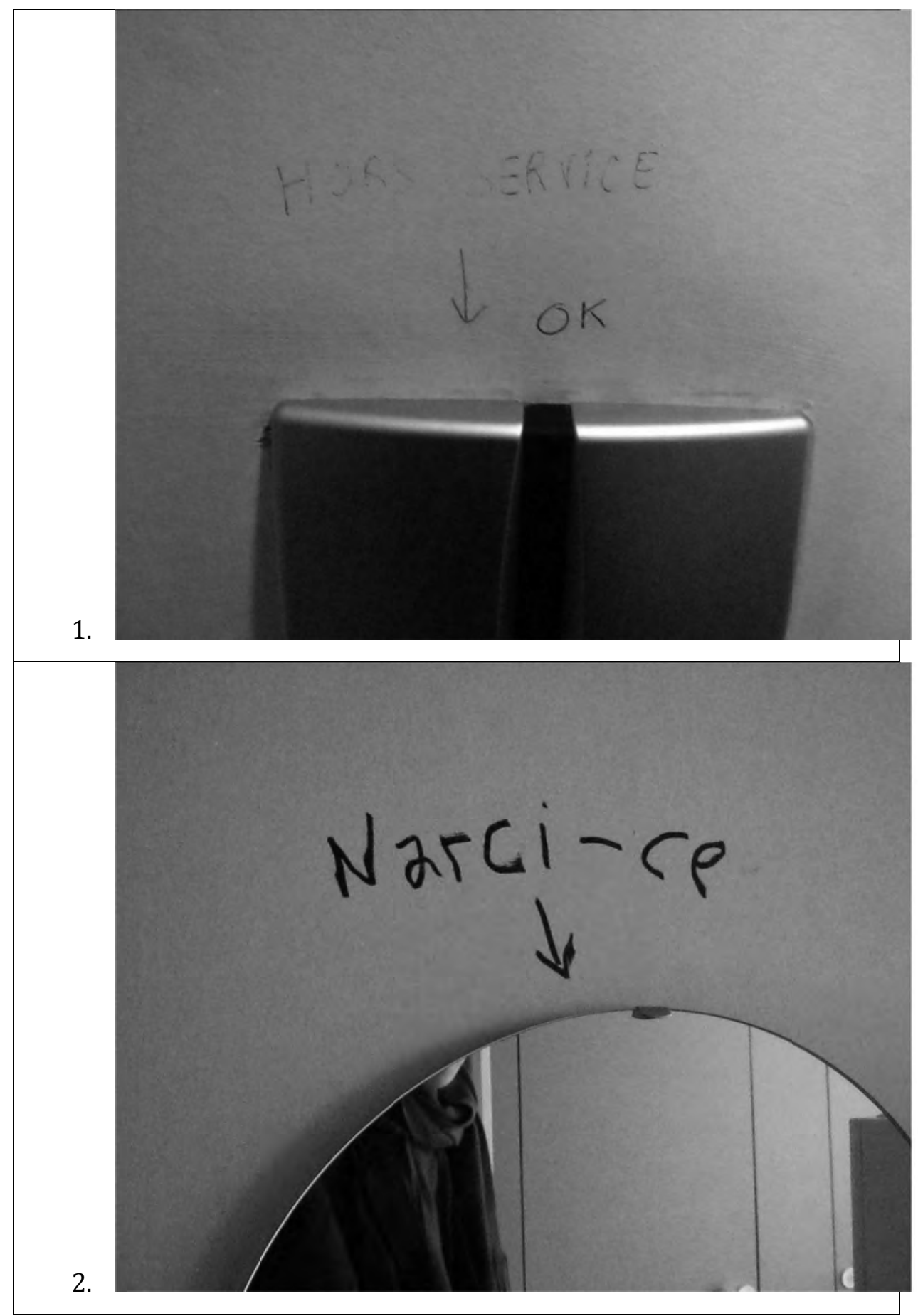

112 


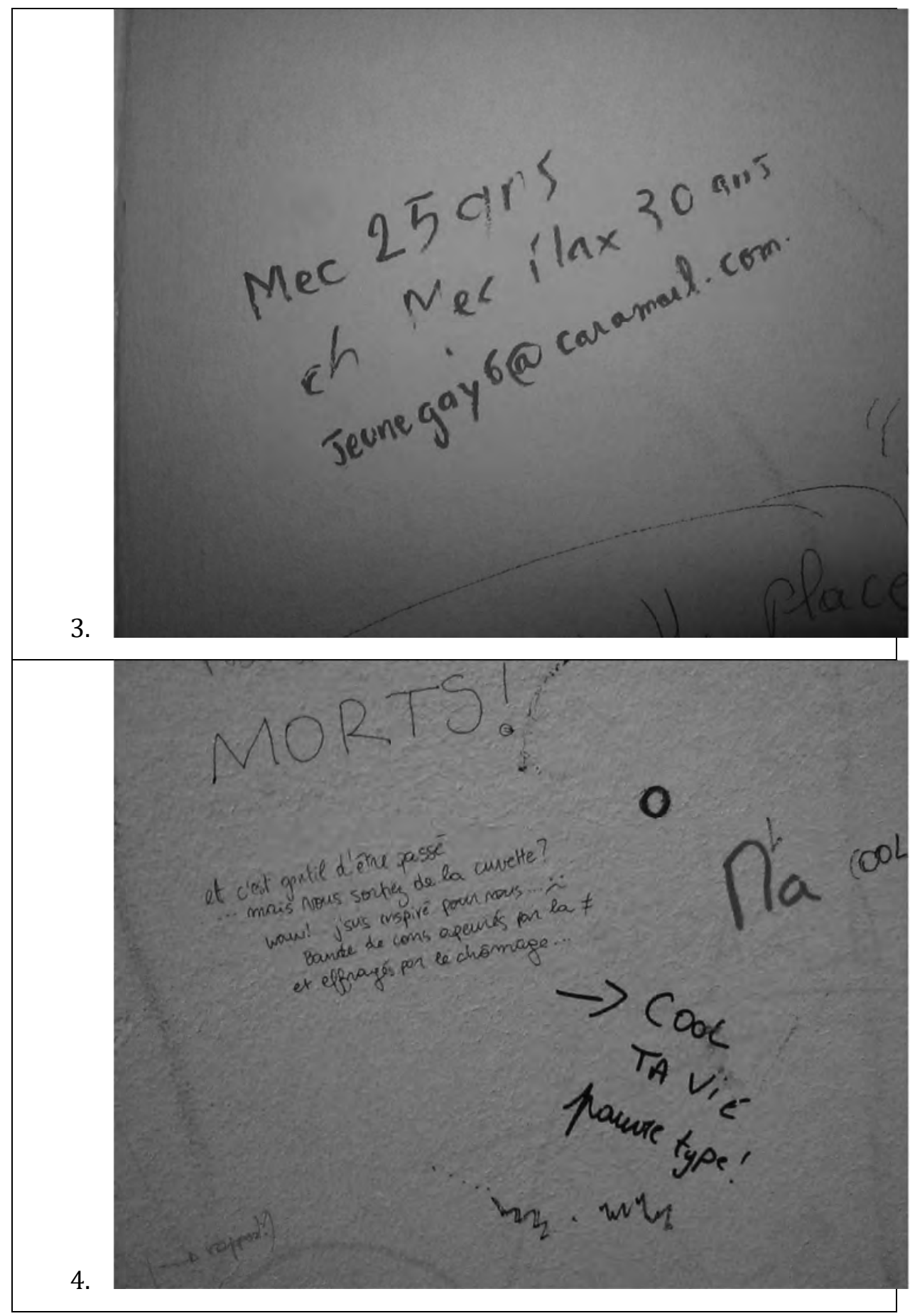




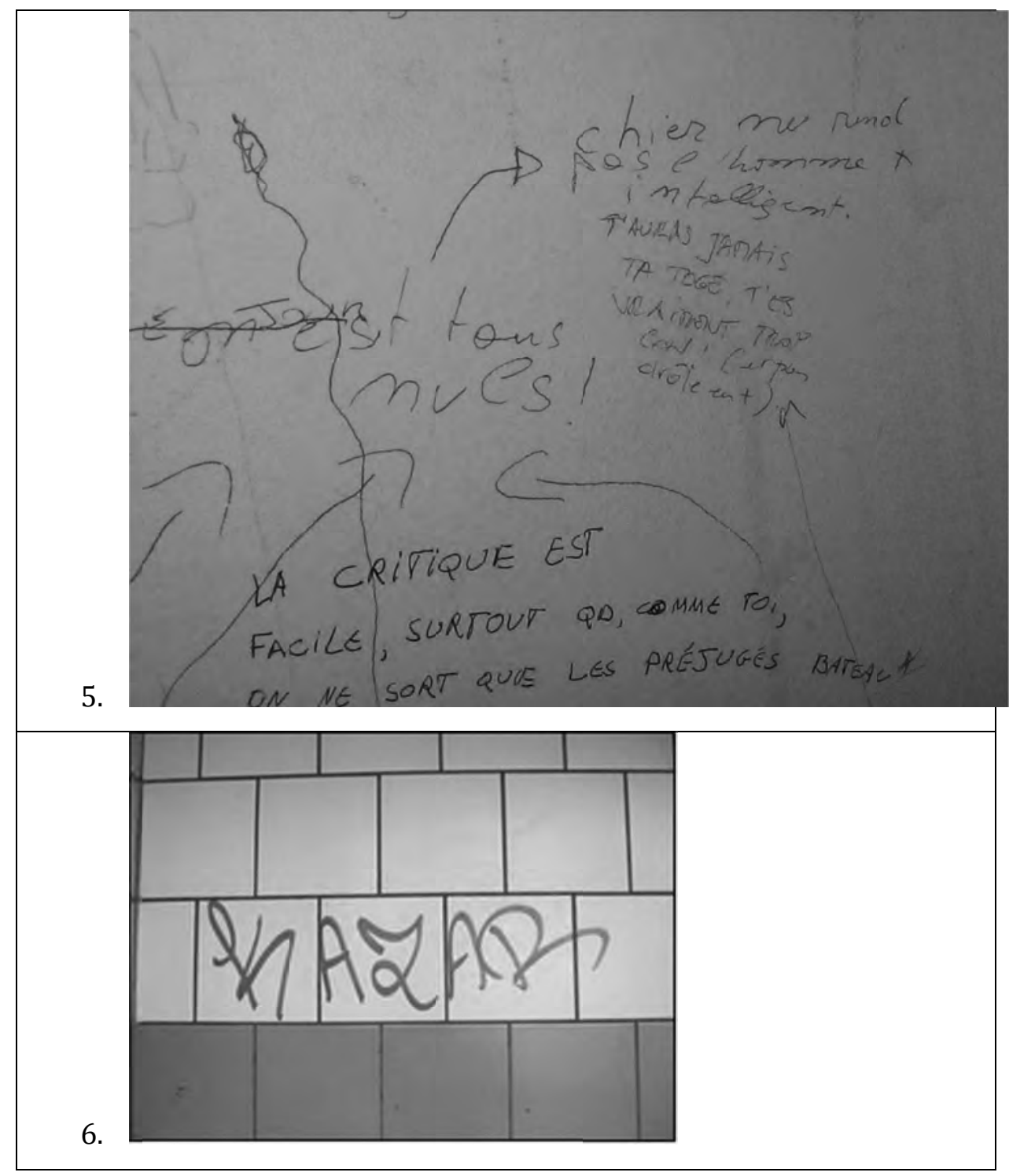




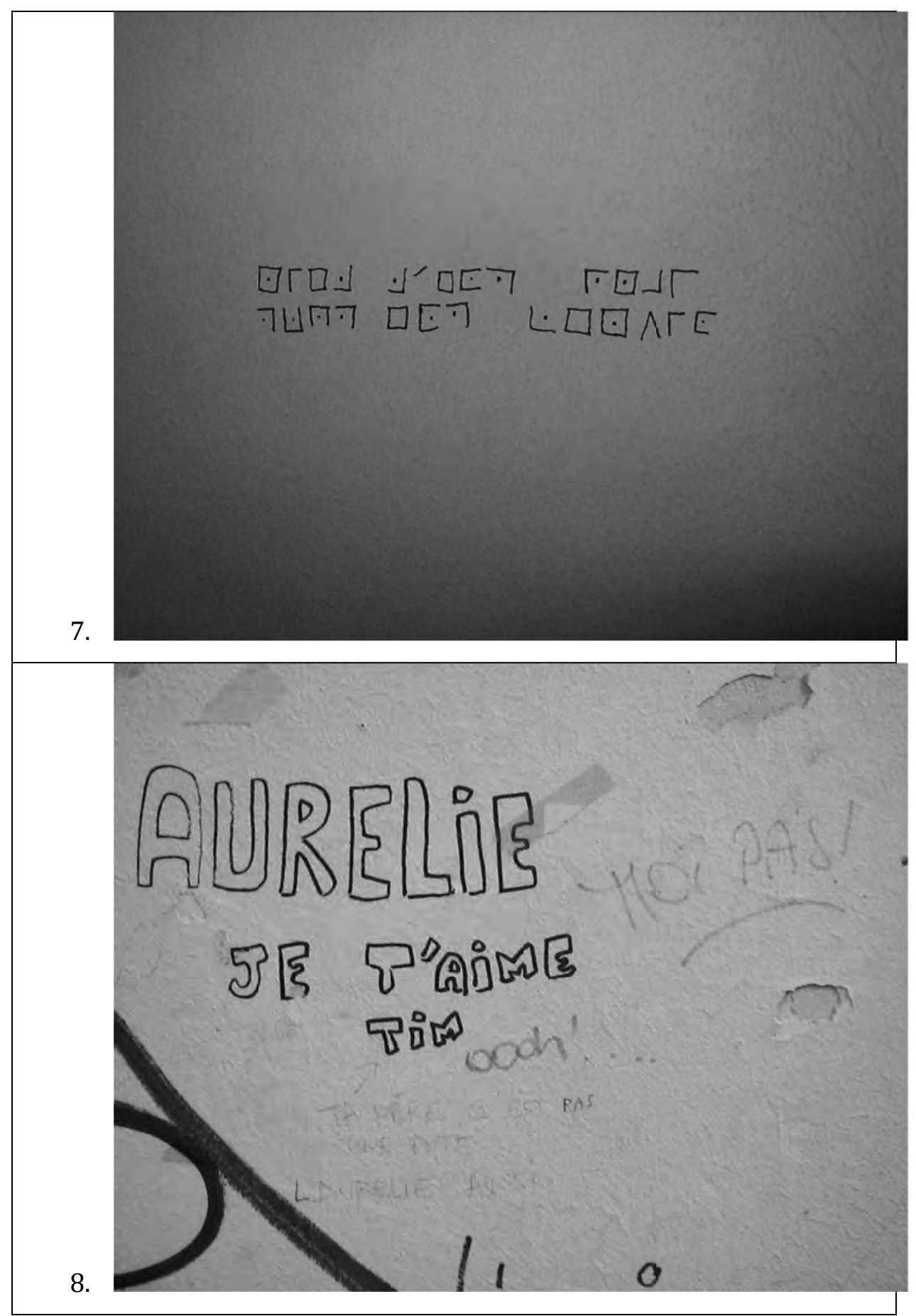




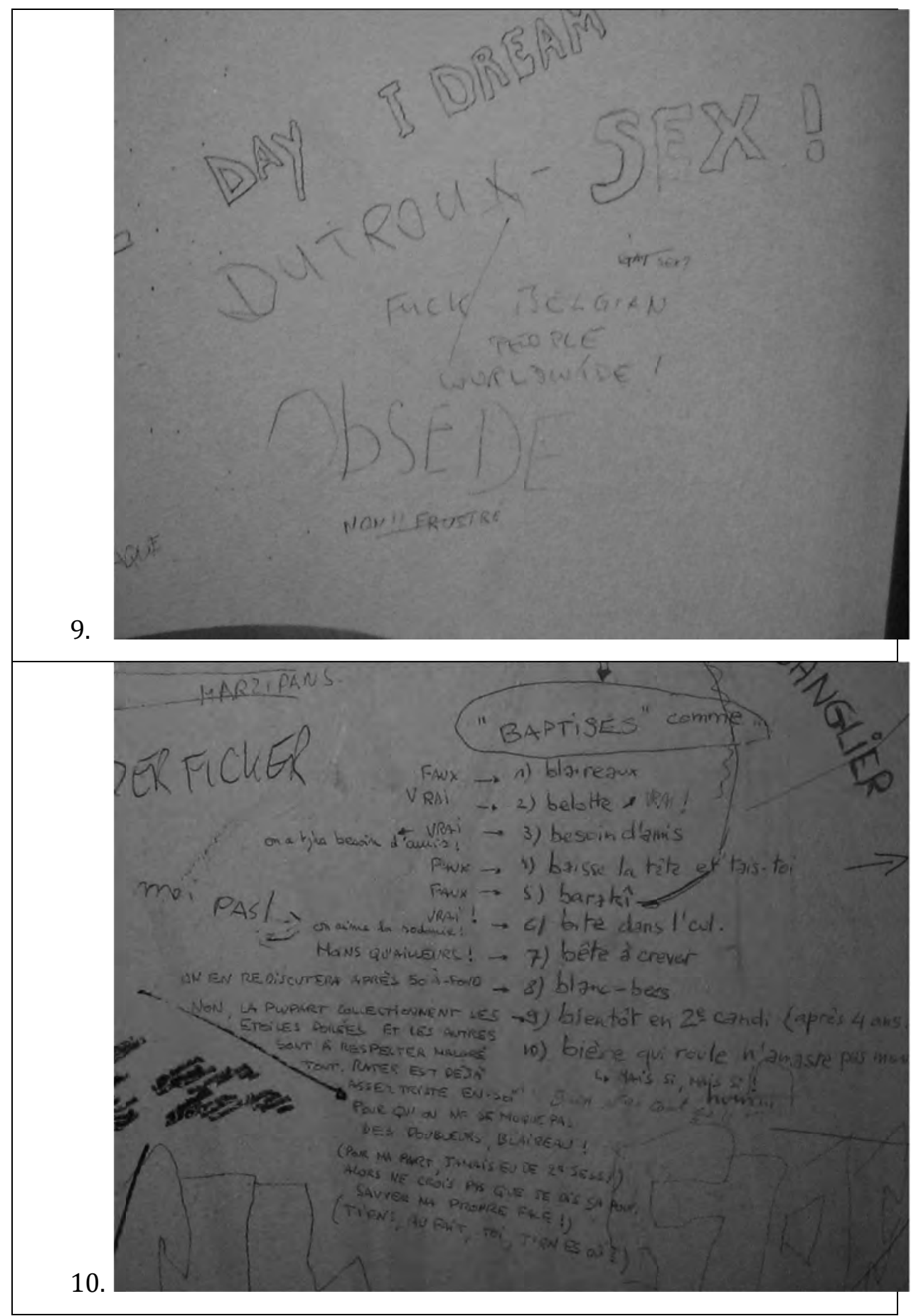




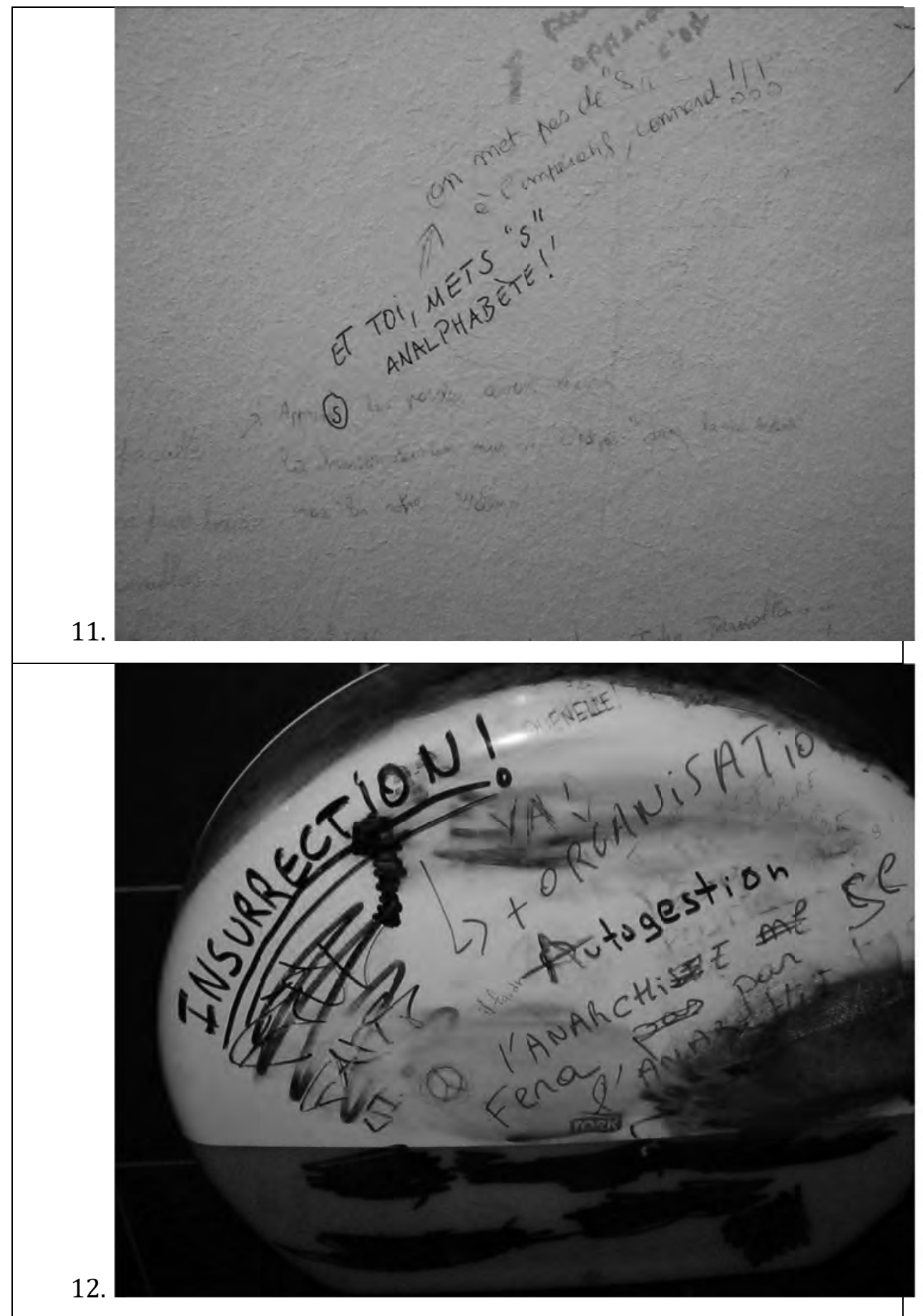




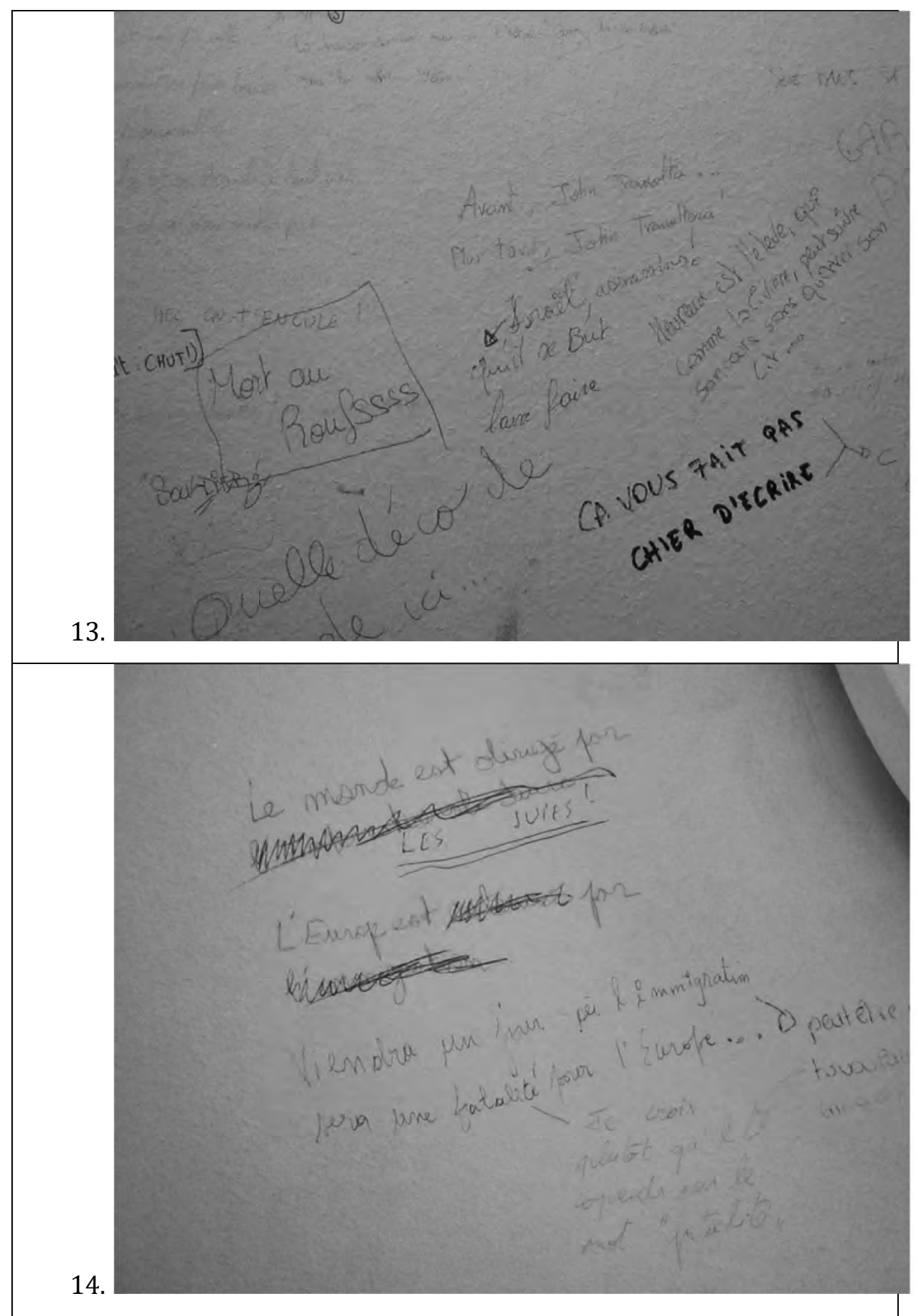




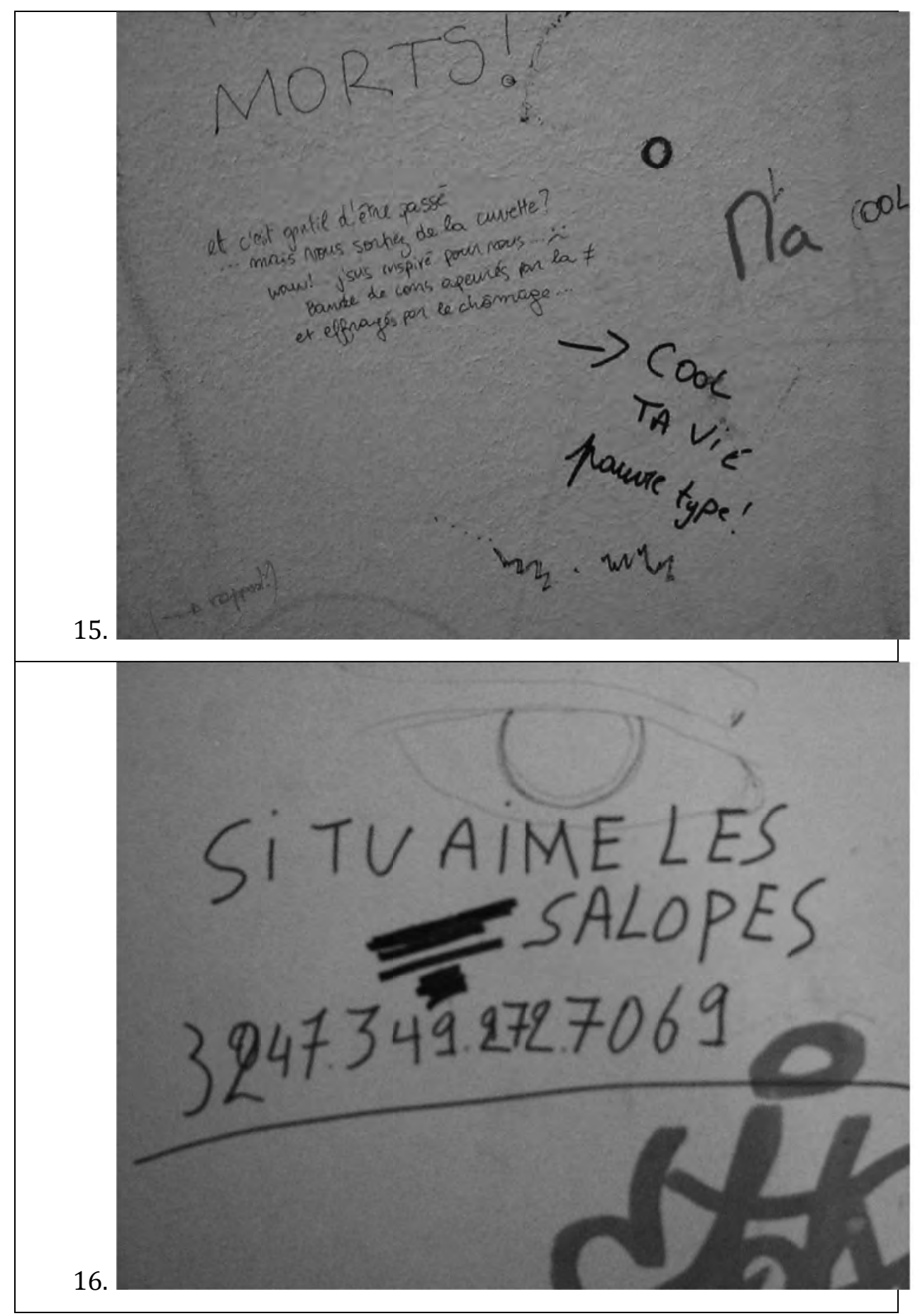




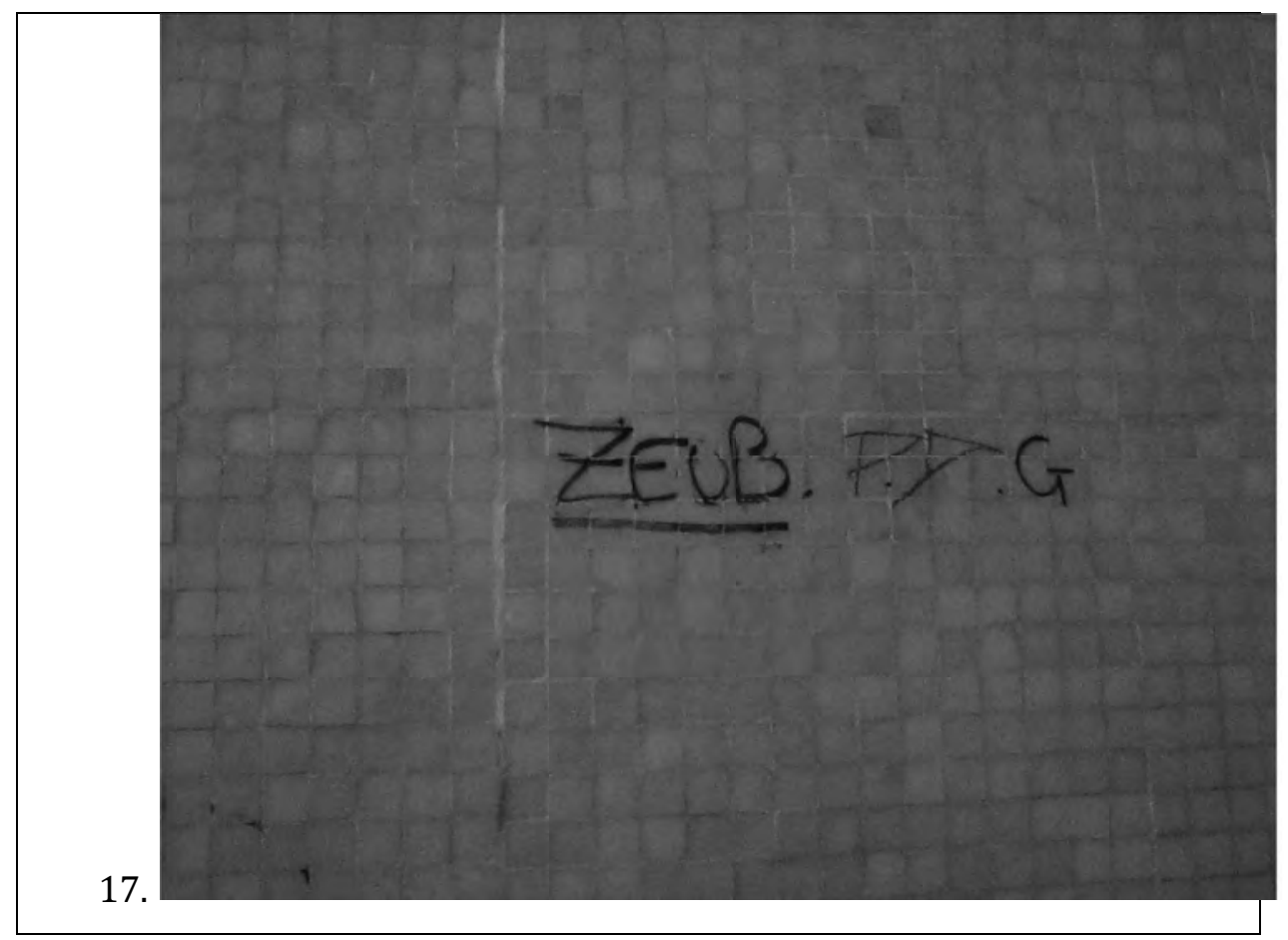

